\title{
Optimization Model and Solutions for punching Machine
}

\author{
Baocheng Wan, Cong Xiao, Taiyang Niu, Tiane Wang \\ Information Technology College, Jilin Agricultural University, Changchun, 130118, China
}

Keywords: optimization scheme; 0-1 programming; AMPL. Cutting tool transformation; Chinese library classification number: literature identification code: article number:

\begin{abstract}
The conversion time of punching machine tool is the important factors influencing the punching machine production efficiency, it increase the efficiency of the punching machine production. In order to solve the same hole type, it needs many and order limitation cutting tools to translate. We establish the knife tool 0-1 programming model of optimal conversion at shortest time and use $0-1$ indicator variables related theory. At the same time we solve logic or relationship in the model, in order to have new models which are easier for computer to process. Making use of the AMPL program software code implementation, we combined the data of 2012 "Shenzhen Cup" national college students' mathematical modeling in the summer camp D and obtain the good effect of the optimal transformation scheme of punching machine tools.
\end{abstract}

\section{Introduction}

Punching machines are mainly used for drilling operation in the manufacture process of printed circuit board, a hole processing costs usually accounted for plate-making fee of $30 \% \sim 40 \%$. How to quickly response to market demand and improve the efficiency of punching machines production have become an important problem needed to solve for manufacturing enterprises who want to improve their competitive ability. Punching machine bit installed on several kinds of cutting tools, different holes need different cutting tool types and processing order. When the punching machine are working, conversion tool cost occupies very large proportion in the whole process of production and processing. And the bit of travel time and tool conversion time are the two factors influencing the production efficiency. The conversion time between two knives is larger than bit on the printed circuit board by the distance between any two furthest holes, so the conversion time of the cutting tool becomes the important factors influencing the punching machine production efficiency. Currently, the research to improve the productivity efficiency of the punching machines has many and mature. Through the various research, the cutting tool transformation scheme for the production efficiency of punching machines is still blank. Therefore, using the optimization theory and modern optimization algorithms on the punching machine drill tool conversion plan is helpful for enterprises to improve production efficiency and reduce the production cost significantly.

In this paper, we solve the problem of the optimal conversion punching machine tool. Regard real life as the background, we use the optimization theory and 0-1 programming knowledge to set up a shortest time of $0-1$ programming model of cutting tool optimal conversion. According to the theory and logic of model and theory of $0-1$ indicator variables (see section to prepare knowledge) that are summarized by us. We improve the original model, so it can be processed by computer. After it, we make use of data of 2012 "Shenzhen cup" national college students mathematical of the summer camp D to experiment. And we use AMPL software to program and solve the problem. Finally, we have optical method of punching machine tool, which shows good effect.

\section{Problem Analysis}

The travel of the bit time and conversion of the cutting tool can influence the production efficiency, when punching machines are processing. The drill of punching machines have several kinds of cutting tools, printed circuit boards have several kinds of holes needed to processed.

Different hole types need different kinds of cutting tools to process. The conversion time between two knives larger the distance between any farthest two holes on the circuit board, in order to 
reduce the production cost and enhance the efficiency of the punch, under the situation of ensure the use of the tool order, we reduce the number of changing knives and reduce the whole time of changing, so we can find a best transformation scheme, which costs shortest time.

Different cutting tools processed different holes, there are limitations of needs of cutting tools and processing order for different holes. In the case of some holes need two or more kinds of cutting tools and order of processed, we adopt the thought of division, which will splits a tool into two knives that is namely as the ideas of the dummy knives. So we can solve the problem of the optimal conversion punching machine tools.

\section{Preliminaries}

We want to distinguish the state $\mathrm{x}=0$ and the state $\mathrm{x}>0$ by using indicator variable $\sigma$. We use $\sigma=1$ indicating $x>0$ or $\sigma=0$ indicating $x \geq 0$. “ $\rightarrow$ ” is used as contain connectors, “ $\leftrightarrow$ ” is used as equivalent connectors.

The related symbols which are involved are defined as follows:

$f(x)$ : any multivariate function;

$\delta$ : instructions variables $0-1$, $\mathrm{t}$ is used to indicate status;

$M$ : the upper bound of $f(x), M$ is large enough;

$m$ : the lower bound of $f(x), m$ is small enough;

$\varepsilon$ : tolerance of $f(x)$;

$L: \inf |f(x)| \neq 0$, if $L>0$, randomly take a positive number $\varepsilon$ less than or equal to $L$, at this time $|f(x)|<\varepsilon \Leftrightarrow f(x)=0$; Otherwise $L=0$, no matter what value of $\varepsilon$ is taken, $|f(x)|<$ $\varepsilon \Leftrightarrow f(x)=0$ will not established.

The following two related theorems can solve the problem effectively.

Theorem 1 If $\delta=1$, the necessary and sufficient condition of $f(x)>0$ is $f(x) \geq m(1-$ $\delta)+\varepsilon \delta$.

Proof. If $\delta=1, f(x)>0$, then $f(x) \geq \varepsilon=m(1-\delta)+\varepsilon \delta$. If $\delta=0, f(x)>0$, then $f(x) \geq m=m(1-\delta)+\varepsilon \delta$. If $\delta=0, f(x) \leq 0$, then $f(x) \geq m=m(1-\delta)+\varepsilon \delta$.

If $\delta=1, f(x) \geq m(1-\delta)+\varepsilon \delta$, then $f(x) \geq \varepsilon$, and then $f(x)>0$.

Theorem 2 If $\delta=1$, the necessary and sufficient condition of $f(x) \geq 0$ is $f(x) \geq m(1-\delta)$.

\section{Models}

This paper involves the related symbols which are defined as follows:

$i$ : denote the first tool, $i=1,2, \ldots, n+2$

$j$ : it means the optimal transformation scheme location of cuttings, $j=1,2, \ldots, n+2$

$n$ : the bit of single drill hole punch of punching tools has $n$ kinds of cutting tools

$o_{i}$ : denote the $i$ cutting number in the orders

$o_{i+1}$ : denote the $i+1$ cutting number in the orders

$p_{j}$ : denote the $j$ cutting number in the orders

$x_{i j}$ : one of the values of two variables, denote if the $i$ tool is on the $j$ position (yes is1, otherwise 0)

Cutting tools of single bit punching machine take lager transformations time than the two holes of longest distance on the printed circuit board, therefore, based on the purpose of taking the shortest time, we want to get optimal operation line of the cutting tools and make tools changes as little as possible.

Different cutting tools processes different holes, different holes have the limitations of the demands about tools and processing order. But in the paper, $\mathrm{E}$ and $\mathrm{F}$ need the same cutting tools $\mathrm{c}$ and $\mathrm{f}$ to do, the processed order is not same. For this situation, we take the mind of a cutting tool splitting into two knives, guaranteeing the processed order of cutting tools, we premise two tools $c^{\prime}$, $f^{\prime}$ as 9 and 10 kinds of cutting tools according to the original order. So we have $n+2$ cutting tools 
to process. The objective function can be represented as:

$\min \sum_{i=1}^{n+1} 4-|4-| o_{i}-o_{i+1}||$

Need to satisfy the constraint conditions:

1) in the optimal transformation scheme, one position has only one tool, i.e.

$\sum_{j=1}^{n} x_{i j}=1, \forall i=1,2, \ldots, n+2$.

2) when it processing through hole, only one tool to process, namely a through hole can't be processed by two tools at same time, i.e.

$\sum_{i=1}^{n} x_{i j}=1, \forall i=1,2, \ldots, n+2$.

3) cutting tool number of $i$ in the order, i.e.

$o_{i}=3 x_{i 9}+6 x_{i 10}+\sum_{j=1}^{n} j x_{i j}, \forall i=1,2, \ldots, n+2$.

4) denote the location number of $j$ in the order of cutting tools, i.e.

$p_{j}=\sum_{i=1}^{n} i x_{i j}, \forall j=1,2 \ldots, n+2$.

5) According to the problem of different holes need different cutting tools and processing order, we establish the following constraints. a cutting tool needs to be used before c tools or c' tools. C tool needs to be used in front of the f tool or f' tool, or c' tool needs to be used before $f$ tool or f' tool, $\mathrm{d}$ tool needs to be used before $\mathrm{g}$ tool. $\mathrm{g}$ tool needs to be used before $\mathrm{f}$ tool or c' tool. e tool needs to be used before c tool or c' tool. c tool needs to be used after the f tool or f' tool, or c' tool needs to be used after $\mathrm{f}$ or $\mathrm{f}$ ', i.e.

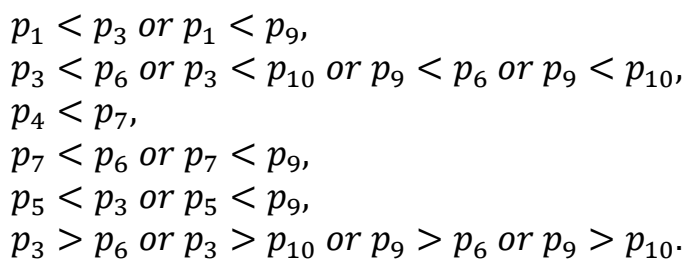

If the relationships in the model can not be directly processed in the program, we can set up the basic principles of establishing 0-1 variables and theorem and deal with constraint conditions.so we can have a better application to solve the model. We usually use a model of two value variable to solve the model choosing of whether to do a certain thing.

Introduce the 0-1 variable linear programming (or sometimes introduce integer programming) as indicators of decision variable or variables. Such variables may contain the $0-1$ variable linear constraint conditions which denote the different decisions or logical relationship. In logical relationship, if everything is true at the same time, the result is true. If the cases are true and false, the result is still true. Only when all are false, the result is false, that is $\sum \delta_{i} \geq 1$.

In applications, taking condition of $\delta=1 \rightarrow f(x)=0$ has another representation which is $f(x)>0 \rightarrow \delta>1$. Combined them as condition of $\delta=1 \rightarrow f(x)>m$ which is denoted $\delta=1$. And $f(x)>0$ can be corrected as $\delta=1 \leftrightarrow f(x)>m$. So according to the theorem 1 , the condition of $\delta=1 \leftrightarrow f(x)>m$ can be denoted as $f(x)-m(1-\delta) \geq 0$. In 0-1 programming, $\varepsilon$ is the tolerance of $f(x)$, i.e., can be denoted as $f(x)-m(1-\delta)-\varepsilon \delta \geq 0$.

That is $p_{1}<p_{3}$, we can use $p_{3}-p_{2}>-(n+2)\left(1-\delta_{1}\right)$. The same to $p_{1}>p_{9}$, it can use $p_{9}-p_{1}>-(n+2)\left(1-\delta_{2}\right)$. So $p_{1}<p_{3}$ or $p_{1}<p_{9}$ can be denoted as :

$$
p_{3}-p_{2}>-(n+2)\left(1-\delta_{1}\right), p_{9}-p_{1}>-(n+2)\left(1-\delta_{2}\right) \text {. }
$$

The rest can be transformed to the inequalities like above. The improved model is raised as follows: 


$$
\begin{aligned}
\min & \sum_{i=1}^{n+1} 4-|4-| o_{i}-o_{i+1}|| \\
\text { s.t. } & \sum_{j=1}^{n} x_{i j}=1, i=1,2, \ldots, n+2, \\
& \sum_{i=1}^{n+2} x_{i j}=1, j=1,2, \ldots, n+2, \\
& o_{i}=3 x_{i 9}+6 x_{i 10}+\sum_{j=1}^{n} j x_{i j}, i=1,2, \ldots, n+2, \\
& p_{j}=\sum_{i=1}^{n} i x_{i j}, j=1,2, \ldots, n+2, \\
& p_{3}-p_{1}>-(n+2)\left(1-\delta_{1}\right), p_{9}-p_{1}>-(n+2)\left(1-\delta_{2}\right), \delta_{1}+\delta_{2} \geq 1, \\
& p_{6}-p_{3}>-(n+2)\left(1-\delta_{3}\right), p_{10}-p_{3}>-(n+2)\left(1-\delta_{4}\right), p_{9}-p_{6}>-(n+2)\left(1-\delta_{5}\right), \\
& p_{9}-p_{10}>-(n+2)\left(1-\delta_{6}\right), \delta_{3}+\delta_{4}+\delta_{5}+\delta_{6} \geq 1, \\
& p_{4}<p_{7}, \\
& p_{6}-p_{7}>-(n+2)\left(1-\delta_{7}\right), p_{10}-p_{7}>-(n+2)\left(1-\delta_{8}\right), \delta_{7}+\delta_{8} \geq 1, \\
& p_{3}-p_{5}>-(n+2)\left(1-\delta_{9}\right), p_{9}-p_{5}>-(n+2)\left(1-\delta_{10}\right), \delta_{9}+\delta_{10} \geq 1, \\
& p_{3}-p_{6}>-(n+2)\left(1-\delta_{11}\right), p_{3}-p_{10}>-(n+2)\left(1-\delta_{12}\right), p_{9}-p_{6} \\
& >-(n+2)\left(1-\delta_{13}\right), \\
& p_{9}-p_{10}>-(n+2)\left(1-\delta_{14}\right), \delta_{11}+\delta_{12}+\delta_{13}+\delta_{14} \geq 1, \\
& x_{i j} \in\{0,1\}, i, j=1,2, \ldots, n+2 .
\end{aligned}
$$

\section{Conclusion}

Combined with the data of 2012 "Shenzhen cup" national college students' mathematical modeling in a summer camp D. Table 1 lists the ten kinds of pass the required processing cutting tool and machining sequence (the * said there is no limitation of cutting tool processing order for the hole types.)

Table1: ten kinds of holes required processing cutting tool and machining sequence

\begin{tabular}{lcccccccccc}
\hline Hole type & A & B & C & D & E & F & G & H & I & J \\
\hline Requiried cutting tool & a & b & a, c & d, e* & c, $f$ & $g, h^{*}$ & $d, g, f$ & $h$ & $e, c$ & $f, c$ \\
\hline
\end{tabular}

For each hole requires tool types in table 1, we give according to alphabetical 8 kinds of cutting tools in turn number.The corresponding cutting tools and cutting tools of numbers are shown in table 2 .

Table 2: the digital number of corresponding cutting tools and cutting tools

\begin{tabular}{lllllllll}
\hline Cutting tool & a & b & c & d & e & f & g & h \\
\hline Serial number & 1 & 2 & 3 & 4 & 5 & 6 & 7 & 8 \\
\hline
\end{tabular}

According to the established 0-1 programming model, we use AMPL software to program. The cutter optimal sequence transformation scheme is $4,3,2,1,8,7,6,5,3$.

In addition, the $0-1$ programming is an effect method to solve a practical problem, the computed results are accurate and high efficiency. But there are also some shortcomings. This model can also be extended to more general increase the efficiency of the punching machine production, and solve the problem of punching machine tools of the optimal conversion example. To a certain extent, it has high practical significance. 


\section{Reference}

[1] WU Ruxue. The improvement of punching machine production efficiency. Guangxi university, 2012-10.

[2] Wang Hua, Wang Tao, Zhao Chenghui. Punching machine operation optimization model and solving. Journal of trait national normal university, 2013.

[3] Lin Bo-liang, Zhu Songnian. Optimization of marshalling plan of nonlinear 0-1 programming model and simulated annealing algorithm. Journal of railway (Chengdu), 1994-6.

[4] 2012 "Shenzhen cup" national college students' mathematical modeling summer camp D problem.

[5] http://ampl.com/ H.P.

[6] [British], Williams Meng Guobi. Mathematical programming model is established and computer application. National defense industry press [Beijing], 1991-6.

[7] Geng Suyun, Qu WanLing, etc. Discrete mathematics (second edition). Tsinghua university press [Beijing], 1999-3-4.

[8] Cathy. Increase the efficiency of the punching machine production plan design. Journal of southwest normal university (natural science edition), 2013-4.

[9] Chen Xue-zhong, Zhao-Han sheng, Li Wenxi. Choose 0-1 goal programming model of scientific research project. The scientific research management, 2005-7.

[10] Ying-hong li. Sort of solution for linear 0-1 programming model. Journal of southwest Jiao Tong university, 2001-10. 